\title{
EXPLICIT MODEL FOR BENDING EDGE WAVE ON AN ELASTIC ORTHOTROPIC PLATE SUPPORTED BY THE WINKLER-FUSS FOUNDATION
}

\author{
S.N. ALTHOBAITI, A. NIKONOV, AND D. PRIKAZCHIKOV
}

\begin{abstract}
The paper is concerned with a bending edge wave on a thin orthotropic elastic plate resting on the Winkler-Fuss foundation. The main focus of the contribution is on derivation of a specialised reduced model accounting for the contribution of the bending edge wave to the overall dynamic response, allowing simplified analysis for a number of dynamic problems. The developed formulation includes an elliptic equation associated with decay over the interior, and a beam-like equation on the edge governing wave propagation accounting for both bending moment and modified shear force excitation, thus highlighting a dual parabolic-elliptic nature of the bending edge wave. A model example illustrates the benefits of the approach.
\end{abstract}

\section{INTRODUCTION}

Bending edge waves have a remarkable history, starting from the original contribution [1], with a preceding analysis of stability within static framework [2], for more details see [3], and a review article [4]. The applications of edge waves are often related to non-destructive testing in engineering structures, e.g. turbine blades etc., see [5], as well as more recent contributions [6],[7], and references therein. We also mention related applications in acoustics, see [8].

Bending edge waves have been mostly studied on an elastic plate subject to homogeneous boundary conditions, see e.g. [9], [10] and [11] accounting for the effect of particular types of anisotropy, as well as a challenging scenario of arbitrary anistropy tackled by means of Stroh formalism, see [12]. We also mention a related problem of instability of plate edges by means of pre-stress, analysed in [13]. Localised waves have also been studied on the interface between two Kirchhoff plates [14], and also on the edge of a circular disk [15].

In addition, we cite the considerations within the framework of 3D elasticity, including that for mixed boundary conditions [16], as well as nontrivial analysis for classical boundary conditions [17], [18].

Other recent developments include studies of bending edge waves on plates supported by elastic foundations, see [19] and [20], as well as plates with the reinforced edge, see [21], [22], and a recent work [23].

The considerations of general forced conditions on the edge are relatively seldom, e.g. within the framework of explicit models for edge waves. We are mentioning some preliminary ideas in [24], and a parabolic-elliptic model developed in [25], with the methodology summarised for Rayleigh and Rayleigh-type waves in [26]. The formulation for the bending edge wave relies on the eigensolution in terms of a single plane harmonic function. This eigensolution is then

Key words and phrases. elastic, plate, bending, edge wave, explicit model.

Althobaiti acknowledges support from Project number (TURSP-2020/305), Taif University, Taif, Saudi Arabia. Nikonov gratefully acknowledges the financial support from the Slovenian Research Agency (ARRS), Grant Ref. J2-9224. Prikazchikov acknowledges support by the Ministry of Education and Science of the Republic of Kazakhstan, Grant IRN AP08857255. 
perturbed in slow time. As a result, the decay away from the edge is governed by an elliptic equation, whereas propagation along the edge is described by a beam-like equation with the loading appearing in the right hand side, mirroring a hyperbolic-elliptic formulation for the Rayleigh wave.

In the current paper this methodology of explicit models, capturing the contribution of the localised wave to the overall dynamic response, is developed further for the case of an orthotropic elastic plate on the Winkler-Fuss foundation, subject to prescribed bending moment and modified shear force on the plate's edge. First, a brief derivation of the bending edge wave of arbitrary profile is presented. Then, a perturbation scheme is established. First, the cases of excitation by means of the bending moment or the modified shear force are treated separately. Then a generalised formulation is proposed, accounting for both types of excitation. This provides another step forward in development of parabolic-elliptic model for the bending edge wave, since the vector problem is essentially reduced to a scalar one. Finally, the developed beam-like equation on the edge is implemented for the bending moment of the point impulse form, allowing an elegant solution in terms of elementary functions.

\section{Statement of the PRoblem}

Consider an elastic plate of thickness $2 h$, occupying the region $-\infty<x<\infty, 0 \leq y<\infty$, $0 \leq z \leq 2 h$, resting on a Winkler-Fuss foundation with the associated domain $-\infty<x<\infty$, $0 \leq y<\infty, 2 h \leqslant z<\infty$, see Fig. 1. We note that the term "Winkler-Fuss" is used in order to acknowledge the early contribution of N.Fuss, see also Section 3.1 in [27]. Within this paper, we assume for the sake of simplicity that the coordinate system is chosen to coincide with the principal directions of anisotropy.

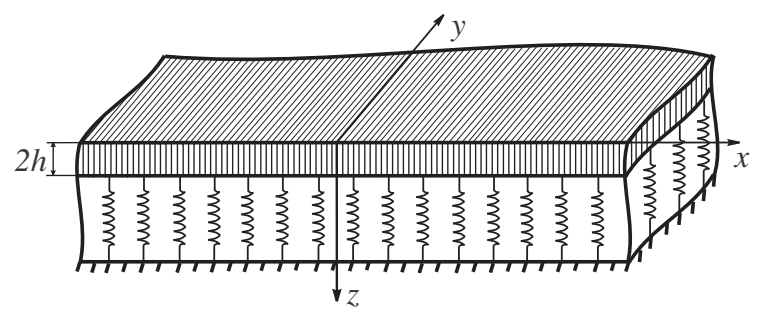

Figure 1. Schematic of an elastic plate on the Winkler-Fuss foundation.

The governing equation for flexural displacement $W$ of an orthotropic, homogeneous, thin elastic plate is written as

$$
\left(\mathcal{L}_{x y}+2 \rho h \partial_{t t}^{2}+\beta\right) W=0,
$$

where the differential operator $\mathcal{L}_{x y}$ is specified as

$$
\mathcal{L}_{x y}=D_{x} \partial_{x x x x}^{4}+2 H \partial_{x x y y}^{4}+D_{y} \partial_{y y y y}^{4},
$$

$\beta$ is the Winkler coefficient, $\rho$ is volume mass density, $D_{x}$ and $D_{y}$ are bending stiffnesses in the $x, y$ directions respectively, with $H=D_{1}+2 D_{x y}$, see [9]. Note that the material parameters must satisfy the conditions ensuring positive definiteness of the strain energy density

$$
D_{x}>0, \quad D_{y}>0, \quad D_{x y}>0, \quad D_{x} D_{y}>D_{1}^{2} .
$$


The boundary conditions at the edge $y=0$ are taken in the form

$$
\begin{aligned}
& \left(D_{1} \partial_{x x}^{2}+D_{y} \partial_{y y}^{2}\right) W=M, \\
& \left(\left(D_{1}+4 D_{x y}\right) \partial_{x x y}^{3}+D_{y} \partial_{y y y}^{3}\right) W=N,
\end{aligned}
$$

where $M=M(x, t)$ and $N=N(x, t)$ are prescribed bending moment and modified shear force, respectively.

\section{Homogeneous WAVE of ARBitrary PRofile}

First, we formulate the eigensolution $(M=N=0)$ for the deflection in terms of a single plane harmonic function, for more detail see [28]. Let us introduce the following dimensionless variables

$$
\xi=\frac{x}{h}, \quad \eta=\frac{y}{h}, \quad \tau=t \sqrt{\frac{D_{x}}{2 \rho h^{5}} .}
$$

Then, the governing equation (1-1) may be rewritten as

$$
\left(\mathcal{L}_{\xi \eta}+\partial_{\tau \tau}^{2}+\beta_{1}\right) W=0
$$

where $\beta_{1}=\beta h^{4} / D_{x}$, with the differential operator $\mathcal{L}_{\xi \eta}$ defined by

$$
\mathcal{L}_{\xi \eta}=\partial_{\xi \xi \xi \xi}^{4}+\frac{2 H}{D_{x}} \partial_{\xi \xi \eta \eta}^{4}+\frac{D_{y}}{D_{x}} \partial_{\eta \eta \eta \eta}^{4} .
$$

Next we adopt the beam-like assumption,

$$
\mathcal{A}_{\tau}[W]=\left(c^{4} \partial_{\xi \xi \xi \xi}^{4}+\partial_{\tau \tau}^{2}+\beta_{1}\right) W=0,
$$

see [2] for more detail, where

$$
c^{4}=1-\frac{\left(\sqrt{4 D_{x y}^{2}+D_{1}^{2}}-2 D_{x y}\right)^{2}}{D_{x} D_{y}}
$$

is a well-known constant first derived in [9], appearing in the dispersion relation for the studied bending edge wave [28]

$$
D_{x} k^{4} c^{4}=2 \rho h \omega^{2}-\beta .
$$

It is emphasized that due to the assumption (2-4) it is possible to transform equation (2-2) to a form that does not involve time derivatives in explicit form, i.e.

$$
\left(D_{y} \partial_{\eta \eta \eta \eta}^{4}+2 H \partial_{\eta \eta \xi \xi}^{4}+D_{x}\left(1-c^{4}\right) \partial_{\xi \xi \xi \xi}^{4}\right) W=0 .
$$

The latter may be shown to be of elliptic type, allowing the operator factorisation

$$
\Delta_{1} \Delta_{2} W=0
$$

where

with

$$
\Delta_{j}=\partial_{\eta \eta}^{2}+\lambda_{j}^{2} \partial_{\xi \xi}^{2}, \quad j=1,2
$$

$$
\lambda_{1}^{2}+\lambda_{2}^{2}=\frac{2 H}{D_{y}}, \quad \lambda_{1}^{2} \lambda_{2}^{2}=\frac{D_{x}}{D_{y}}\left(1-c^{4}\right) .
$$

Below we focus on the scenario when $\lambda_{j}>0, j=1,2$.

It should be noted that the dispersion relation of the elastically supported beam governed by (2-4) coincides with the dispersion relation (2-6) of the bending edge wave under consideration. It is also emphasized that the assumption (2-4) is essentially mirroring the representation of the 
eigensolution of arbitrary profile for Rayleigh waves in the form $f(x \pm c t, y)$, which may be also expressed implicitly as $\left(\partial_{t t}^{2}-c^{2} \partial_{x x}^{2}\right) f=0$, for more detail see [26].

The deflection $W$ may now be expressed as a sum of two arbitrary functions, harmonic in the first two arguments (and decaying as $y \rightarrow \infty$ ), namely

$$
W=\sum_{j=1}^{2} W_{j}\left(\xi, \lambda_{j} \eta, \tau\right) .
$$

Note that throughout this paper the summation convention is not employed. Now, on substituting solution (2-11) into the free edge boundary conditions (1-3), when $M=N=0$, and using properties of harmonic functions, we deduce (at $\eta=0$ )

$$
\sum_{j=1}^{2} \mathcal{M}_{j}\left[W_{j}\right]=0, \quad \sum_{j=1}^{2} \mathcal{N}_{j}\left[W_{j}\right]=0,
$$

with the differential operators $\mathcal{M}_{j}$ and $\mathcal{N}_{j}$ defined as

$$
\begin{aligned}
\mathcal{M}_{j} & =\left(D_{1}-\lambda_{j}^{2} D_{y}\right) \partial_{\xi \xi}^{2}, \\
\mathcal{N}_{j} & =\lambda_{j}\left(D_{1}+4 D_{x y}-\lambda_{j}^{2} D_{y}\right) \partial_{\xi \xi \xi}^{3} .
\end{aligned}
$$

It may be observed that the solvability of (2-12) leads to the dispersion relation (2-6), with $c$ defined by formula (2-5).

Moreover, a relation between the harmonic functions $W_{j}$ may be inferred from equations (2-12), from which the deflection $W$ is expressed through $W_{1}$ as

$$
W=W_{1}\left(\xi, \lambda_{1} \eta, \tau\right)+\theta W_{1}\left(\xi, \lambda_{2} \zeta, \tau\right)
$$

where

$$
\theta=\frac{\lambda_{1}^{2} D_{y}-D_{1}}{D_{1}-\lambda_{2}^{2} D_{y}}
$$

\section{Explicit MOdEL For THE BENDING EDGE WAVE}

Let us now derive the specialised formulation for contribution of the bending edge wave to the overall dynamic response. Following [25], an asymptotic procedure perturbing the eigensolution of Section 2 in slow time is established. Let us introduce the fast $\left(\tau_{f}=\tau\right)$ and slow $\left(\tau_{s}=\epsilon \tau\right)$ time variables accordingly, where $\epsilon \ll 1$ is a small parameter, therefore

$$
\partial_{\tau \tau}^{2}=\partial_{\tau_{f} \tau_{f}}^{2}+2 \epsilon \partial_{\tau_{f} \tau_{s}}^{2}+\epsilon^{2} \partial_{\tau_{s} \tau_{s}}^{2}
$$

It is worth mentioning that the small parameter $\epsilon$ may be associated physically with a small deviation of the phase speed of the analysed motion from that of the homogeneous edge wave. Next, the deflection $W$ is expanded as asymptotic series

$$
W=\frac{h^{2} P}{\epsilon D_{x}}\left(W^{(0)}+\epsilon W^{(1)}+\ldots\right)
$$

where

$$
P=\max _{x, t}[M(x, t), h N(x, t)]
$$


3A. Perturbation procedure for the governing equation of motion. On substituting the asymptotic series (3-2) into the equation of motion (2-2), at leading order we have

$$
\left(\mathcal{L}_{\xi \eta}+\partial_{\tau_{f} \tau_{f}}^{2}+\beta_{1}\right) W^{(0)}=0
$$

Then, in view of the beam-like assumption (see (2-4)),

$$
\mathcal{A}_{\tau_{f}}\left[W^{(0)}\right]=0
$$

equation (3-3) becomes

$$
\Delta_{1} \Delta_{2} W^{(0)}=0
$$

with $\Delta_{j}$ defined in (2-9). Hence, the leading order solution is given by

$$
W^{(0)}=\sum_{j=1}^{2} W_{j}^{(0)}\left(\xi, \lambda_{j} \eta, \tau_{f}, \tau_{s}\right)
$$

where $W_{j},(j=1,2)$ are harmonic functions, decaying at $\zeta \rightarrow \infty$, similarly to (2-11). At next order, using the beam-like assumption

$$
\mathcal{A}_{\tau_{f}}\left[W^{(1)}\right]=0,
$$

and (3-6), we have

$$
\Delta_{1} \Delta_{2} W^{(1)}=-2 \partial_{\tau_{f} \tau_{s}}^{2} W_{1}^{(0)}-2 \partial_{\tau_{f} \tau_{s}}^{2} W_{2}^{(0)}
$$

Then, using the superposition principle, the next order corrector is represented as

$$
W^{(1)}=W_{1}^{(1)}+W_{2}^{(1)}
$$

with $W_{1}^{(1)}$ and $W_{2}^{(1)}$ denoting the particular solutions of (3-7), corresponding to the first and second terms in the R.H.S., respectively.

Now, employing the properties of harmonic functions, it could be shown that

$$
\Delta_{i} W_{j}^{(0)}=\left(\lambda_{i}^{2}-\lambda_{j}^{2}\right) \partial_{\xi \xi}^{2} W_{j}^{(0)}, \quad(1 \leq i \neq j \leq 2) .
$$

Hence, it follows from (3-7) that

$$
\Delta_{j} \partial_{\xi \xi}^{2} W_{j}^{(1)}=(-1)^{j} 2 \kappa \partial_{\tau_{f} \tau_{s}}^{2} W_{j}^{(0)}, \quad j=1,2,
$$

where

$$
\kappa=\frac{1}{\lambda_{2}^{2}-\lambda_{1}^{2}}=\frac{D_{y}}{2 \sqrt{H^{2}-D_{x} D_{y}\left(1-c^{4}\right)}} .
$$

It is convenient to continue the process for the derivatives $\partial_{\xi \xi \eta}^{3} W_{j}^{(1)},(j=1,2)$, for which the solutions of (3-10) may be found in the form

$$
\partial_{\xi \xi \eta}^{3} W_{j}^{(1)}=\partial_{\xi \xi \eta}^{3} U_{j}^{1,0}+(-1)^{j} \kappa \eta \partial_{\tau_{f} \tau_{s}}^{2} W_{j}^{(0)},
$$

where $U_{j}^{(1,0)}=U_{j}^{(1,0)}\left(\xi, \lambda_{j} \eta, \tau_{f}, \tau_{s}\right)$ are arbitrary functions, harmonic in the first two variables. Thus, the two-term solutions of the governing equation of motion (2-2) are written as

$$
\partial_{\xi \xi \eta}^{3} W=\frac{h^{2} P}{\epsilon D_{x}} \sum_{j=1}^{2}\left[\partial_{\xi \xi \eta}^{3}\left(W_{j}^{(0)}+\epsilon U_{j}^{(1,0)}\right)+(-1)^{j} \epsilon \kappa \eta \partial_{\tau_{f} \tau_{s}}^{2} W_{j}^{(0)}+\ldots\right] .
$$


3B. Boundary conditions. Now we are in position to tackle the non-homogeneous boundary conditions (1-3) at $\eta=0$. At leading order we have

$$
\sum_{j=1}^{2} \mathcal{M}_{j}\left[W_{j}^{(0)}\right]=0, \quad \sum_{j=1}^{2} \mathcal{N}_{j}\left[W_{j}^{(0)}\right]=0,
$$

where operators $\mathcal{M}_{j}$ and $\mathcal{N}_{j}$ have been defined in (2-13). Therefore, the functions $W_{j}^{(0)}$ are related through

with $\theta$ defined in (2-15).

$$
W_{2}^{(0)}=\theta W_{1}^{(0)}
$$

Let us now concentrate on the excitation due to bending moment $(M \neq 0, N=0)$. At next order $O(1)$ the boundary conditions (1-3), differentiated twice by $\xi$, yield

$$
\begin{gathered}
\sum_{j=1}^{2}\left(D_{1} \partial_{\xi \xi \xi \xi}^{4}+D_{y} \partial_{\xi \xi \eta \eta}^{4}\right) W_{j}^{(1)}=\frac{D_{x}}{P} \partial_{\xi \xi}^{2} M, \\
\sum_{j=1}^{2}\left(\left(D_{1}+4 D_{x y}\right) \partial_{\xi \xi \xi \xi \eta}^{5}+D_{y} \partial_{\xi \xi \eta \eta \eta}^{5}\right) W_{j}^{(1)}=0 .
\end{gathered}
$$

Note that from (3-10)

$$
\lambda_{j}^{2} \partial_{\xi \xi \xi \xi}^{4} W_{j}^{(1)}=\Delta_{j} \partial_{\xi \xi}^{2} W_{j}^{(1)}-\partial_{\xi \xi \eta \eta}^{4} W_{j}^{(1)}=(-1)^{j} 2 \kappa \partial_{\tau_{f} \tau_{s}}^{2} W_{1}^{(0)}-\partial_{\xi \xi \eta \eta}^{4} W_{j}^{(1)} .
$$

Hence, using (3-13) and (3-17), the boundary conditions (3-16) become

$$
\begin{aligned}
& \sum_{j=1}^{2} \partial_{\xi \xi} \mathcal{M}_{j}\left[U_{j}^{(1,0)}\right]=\frac{D_{x}}{P} \partial_{\xi \xi} M+\kappa \sum_{j=1}^{2}(-1)^{j}\left(D_{y}+D_{1} \lambda_{j}^{-2}\right) \partial_{\tau_{f} \tau_{s}}^{2} W_{1}^{(0)} \\
& \sum_{j=1}^{2} \partial_{\xi} \mathcal{N}_{j}\left[U_{j}^{(1,0)}\right]=2 \kappa D_{y} \sum_{j=1}^{2}(-1)^{j+1} \lambda_{j} \partial_{\tau_{f} \tau_{s}}^{2} W_{j}^{(0)}
\end{aligned}
$$

where harmonic conjugation and integration with respect to $\xi$ was applied to the second equation. Now, employing (3-15) and also the inferred fact that $W^{(0)}=(1+\theta) W_{1}^{(0)}$ on the edge $\eta=0$, after some algebraic manipulations the solvability of (3-18) implies

$$
\partial_{\tau_{f} \tau_{s}}^{2} W^{(0)}=\frac{Q_{M} D_{x}}{2 P} \partial_{\xi \xi}^{2} M
$$

where $Q_{M}$ is a material constant given by

$$
Q_{M}=\frac{\chi\left(\chi+D_{1}\right)}{D_{y}\left(\chi+2 D_{x y}\right)}
$$

with $\chi=\sqrt{D_{x} D_{y}\left(1-\gamma^{4}\right)}$.

Hence, using the beam-like assumption (3-4), along with the leading-order approximation

$$
W \approx \frac{h^{2} P}{\epsilon D_{x}} W^{(0)}
$$

equation (3-19) can be transformed to

$$
\mathcal{A}_{\tau_{f}}[W]+2 \epsilon \partial_{\tau_{f} \tau_{s}}^{2} W=Q_{M} \partial_{\xi \xi}^{2} M
$$


Due to the operator relation

$$
\partial_{\tau_{f} \tau_{f}}^{2}+2 \epsilon \partial_{\tau_{f} \tau_{s}}^{2}=\partial_{\tau \tau}^{2}+O\left(\varepsilon^{2}\right)
$$

on returning to the original coordinates equation (3-22) may be recast as

$$
\mathcal{B}[W]=Q_{M} \partial_{x x}^{2} M,
$$

with the operator $\mathcal{B}$ defined as

$$
\mathcal{B}=D_{x} c^{4} \partial_{x x x x}^{4}+2 \rho h \partial_{t t}^{2}+\beta .
$$

Thus, for the bending edge wave excited by the specified bending moment $M(x, t)$ the deflection of the edge $y=0$ is governed by the beam-like equation (3-23). Note that the results for the isotropic Kirchhoff plate follow immediately from (3-23), provided that the bending stiffnesses $D_{x}=D_{y}=D, D_{1}=\nu D, 2 D_{x y}=(1-\nu) D$ (cf. equation (5.19) in [25]), with the constant $Q$ becoming identical to that defined in formula (5.15) of the cited paper.

The decay over the interior is described by the elliptic equation

$$
\left(\partial_{y y}^{2}+\lambda_{1}^{2} \partial_{x x}^{2}\right) W_{1}=0
$$

with the deflection $W$ found from (2-14). As a result, we have a simpler formulation for a scaled Laplace equation, not a bi-harmonic one. Thus, a dual parabolic-elliptic nature of the studied wave is established, with the solution of the beam-like equation (3-23) acting as a boundary value for the elliptic equation (3-25).

A similar formulation may be derived for the second type of excitation, namely by the modified shear force $N=N\left(x_{1}, t\right)$, now with $M=0, N \neq 0$ in the boundary conditions on the edge $\eta=0$, see (1-3). The procedure is rather similar to that presented in the current subsection. At leading order, once again the boundary conditions (3-14) are revealed, implying the dispersion relation as their solvability condition. At next order, in view of (3-13), it is convenient to differentiate twice with respect to $\xi$, which gives (at $\eta=0$ )

$$
\begin{array}{r}
\sum_{j=1}^{2}\left(D_{1} \partial_{\xi \xi \xi \xi}^{4}+D_{y} \partial_{\xi \xi \eta \eta}^{4}\right) W_{j}^{(1)}=0 \\
\sum_{j=1}^{2}\left(\left(D_{1}+4 D_{x y}\right) \partial_{\xi \xi \xi \xi \eta}^{5}+D_{y} \partial_{\xi \xi \eta \eta \eta}^{5}\right) W_{j}^{(1)}=\frac{h D_{x}}{P} \partial_{\xi \xi}^{2} N .
\end{array}
$$

Substituting (3-13) into (3-26) and following a similar procedure to that presented for the bending moment excitation earlier in this section, it is possible to obtain a beam-like equation on the edge $y=0$, however, this will be written in terms of the rotation angle $V=\partial_{y} W$, namely

$$
\mathcal{B}[V]=-Q_{M} \partial_{x x}^{2} N,
$$

with the constant $Q_{M}$ defined in Eq. (3-20). As follows from Eq. (2-14), on making use of the Cauchy-Riemann identities, the rotation angle on the edge is expressed through a single harmonic function $W_{1}$ as

$$
\left.V\right|_{y=0}=\left.\partial_{y} W\right|_{y=0}=-\left.\left(\lambda_{1}+\theta \lambda_{2}\right) W_{1, x}^{*}\right|_{y=0},
$$

with the asterisk denoting a harmonic conjugate and $\theta$ defined in Eq. (2-15). Therefore, Eq. (3-27) may be rewritten as

$$
\mathcal{B}[W]=Q_{N} \sqrt{-\partial_{x x}^{2}}(N),
$$


where

$$
Q_{N}=-\frac{1+\theta}{\lambda_{1}+\theta \lambda_{2}} Q_{M},
$$

and $\sqrt{-\partial_{x x}^{2}}$ is understood as a pseudo-differential operator, appearing previously in hyperbolicelliptic models for the Rayleigh wave, see e.g. Section 5.1 in [26].

Thus, the explicit model for the combined excitation of the bending edge wave by means of both bending edge moment $M$ and the modified shear force $N$ may be formulated. It is comprised of a beam-like equation

$$
\mathcal{B}[W]=Q_{M} \partial_{x x}^{2} M+Q_{N} \sqrt{-\partial_{x x}^{2}}(N),
$$

with the solution of the latter serving as a boundary value for the elliptic equation (3-25), with the deflection $W$ related to the auxiliary harmonic function $W_{1}$ by $(2-14)$.

\section{Model EXAMPLE}

To illustrate the derived formulation, let us now consider a model example. For the sake of simplicity, assume an unsupported plate, so $\beta=0$. Let the bending edge wave be excited by a point impulse bending moment, i.e.

$$
M=-M_{0} \delta(x) \delta(t) .
$$

Then, the beam-like equation (3-31) on the edge $y=0$ becomes

$$
\left(D_{x} c^{4} \partial_{x x x x}^{4}+2 \rho h \partial_{t t}^{2}\right) W=-M_{0} Q_{M} \delta^{\prime \prime}(x) \delta(t) .
$$

On applying the Fourier and Laplace integral transforms with respect to $x$ and $t$, the transformed deflection $W^{F L}$ on the edge $y=0$ is given by

$$
\left.W^{F L}\right|_{y=0}=\frac{M_{0} Q_{M} p^{2}}{D_{x} c^{4} p^{4}+2 \rho h s^{2}},
$$

where $p$ and $s$ are the parameters of the Fourier and Laplace integral transforms, respectively.

The associated solution of the "exact" problem formulation, including the equation of motion (1-1) subject to the boundary conditions (1-3) with $N=0$ and prescribed edge bending moment (4-1), may be written as

$$
\left.W_{e x}^{F L}\right|_{y=0}=\frac{M_{0}\left(q+D_{1} p^{2}\right) p^{2}}{q^{2}+4 p^{2} D_{x y} q-D_{1}^{2} p^{4}}
$$

where

$$
q=\sqrt{D_{y}\left(D_{x} p^{4}+2 \rho h s^{2}\right)} .
$$

It should be noted that the exact solution (4-4) is less trivial for analysis compared to the asymptotic result (4-3), in particular, involving branch points. At the same time, both exact and approximate solutions have the same poles $D_{x} c^{4} p^{4}+2 \rho h s^{2}=0$ associated with the bending edge wave.

Now, applying inverse Laplace transform to (4-3), we deduce

$$
\left.W^{F}\right|_{y=0}=\frac{M_{0} Q_{M}}{\sqrt{2 \rho h D_{x}} c^{2}} \sin \left(\sqrt{\frac{D_{x}}{2 \rho h}} c^{2} p^{2} t\right) .
$$


Therefore, using a standard integral

$$
\int_{0}^{\infty} \sin \left(a p^{2}\right) \cos (2 b p) d p=\frac{1}{2} \sqrt{\frac{\pi}{a}} \cos \left(\frac{\pi}{4}+\frac{b^{2}}{a}\right),
$$

see [29], the inverse Fourier transform for (4-6) can be computed, giving explicitly for edge deflection

$$
\left.W\right|_{y=0}=\frac{M_{0} Q_{M}}{\sqrt[4]{2 \rho h D_{x}^{3} \pi^{2} t^{2}} c^{3}} \cos \left(\frac{\pi}{4}+\sqrt{\frac{2 \rho h}{D_{x}}} \frac{x^{2}}{4 c^{2} t}\right) .
$$

Let us present a numerical illustration of the obtained solution. Introduce the scaled edge deflection

$$
\tilde{W}=\left.\frac{\sqrt{2 \pi \rho h^{3} D_{x}} c^{2}}{M_{0} Q_{M}} W\right|_{y=0}=\frac{1}{\sqrt{\tau_{1}}} \cos \left(\frac{\pi}{4}+\frac{\xi^{2}}{4 \tau_{1}}\right),
$$

where, as above $x=h \xi$ and the dimensionless time $\tau_{1}$ is defined by

$$
\tau_{1}=\sqrt{\frac{D_{x}}{2 \rho h^{5}}} c^{2} t
$$

Note that (4-9) demonstrates a generic behaviour, independent of material properties, appearing in scaling. The following Fig. 2 shows the profile of the solution for several moments of time, with $\tau_{1}$ taken as $0.1,0.3$ and 1.0, depicted by dotted, dashed and solid lines, respectively. It is visible how the initially fast oscillations smoothen up and disperse with time. Another observation notable from Eq. (4-9) relates to the decay of amplitude as $O\left(\tau_{1}^{-1 / 2}\right)$, which is also confirmed by Fig. 2 .

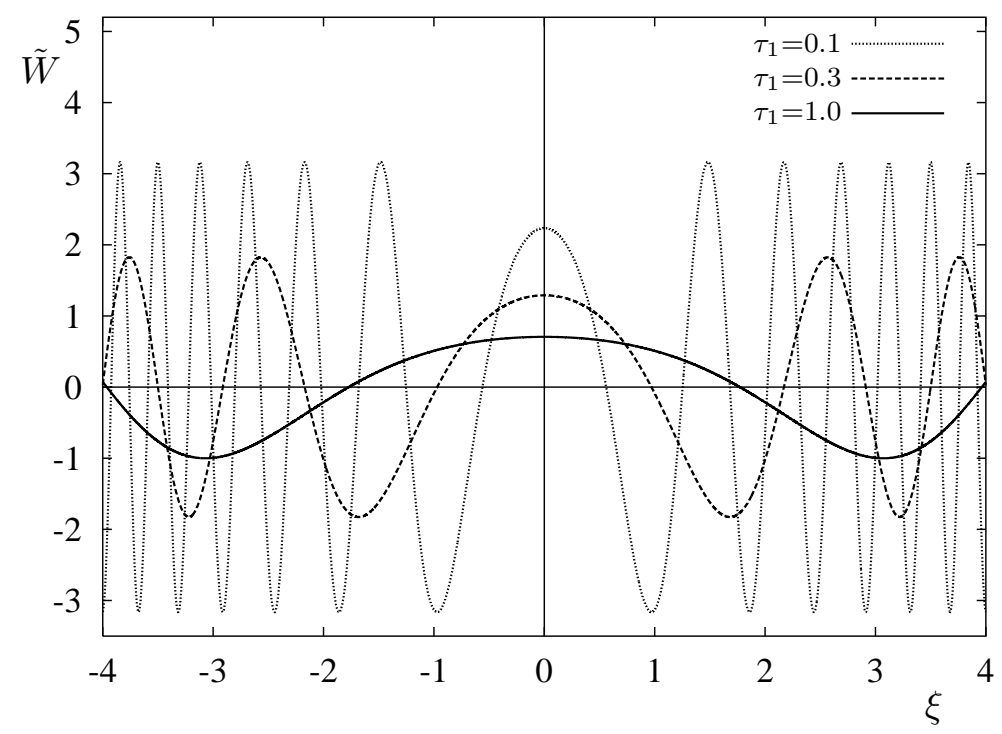

Figure 2. Snapshots of edge deflection (4-9) at $\tau_{1}=0.1 ; 0.3 ; 1.0$. 


\section{Conclusions}

In this paper a parabolic-elliptic explicit model for the bending edge wave on a semi-infinite, orthotropic, elastic plate supported by a Winkler-Fuss foundation, excited by prescribed bending moment and modified shear force, was derived. The formulation contains an elliptic equation (3-25) governing decay away from the edge, and a parabolic beam-like equation (3-31) along the edge, associated with wave propagation. This beam-like equation accounts for both types of loading, achieving further progress compared to previously known separate formulations for the deflection and the angle of rotation, see [25]. The approach is illustrated by a model example, allowing an elegant expression for the Green function for edge deflection in case of excitation by bending moment, see (4-8).

The obtained results develop further the methodology of explicit asymptotic models for Rayleigh and Rayleigh-type waves summarised in [26]. Some parallels of the presented asymptotic procedure with that in [30] for the Rayleigh wave on an orthorhombic half-space may be observed, i.e. related to perturbation scheme for a biharmonic equation. Future plans may involve accounting for more sophisticated foundations, see e.g. [20], treating a plate of arbitrary anisotropy, which will require Stroh formalism in parallel with recent results for the Rayleigh waves [31]. Finally, we mention exciting applications related to elastic plates with resonators, see e.g. [32], and also to topological rainbow effect, see a recent contribution [33].

\section{REFERENCES}

[1] Y. K. Konenkov, "A Rayleigh-type flexural wave", Soviet Physics Acoustics 6 (1960), 122-123.

[2] A. Y. Ishlinsky, "A particular limit transition in the theory of the stability of rectangular elastic plates", Doklady Akademii Nauk SSSR 95:3 (1954), 474-479.

[3] A. N. Norris, V. V. Krylov, and I. D. Abrahams, "Flexural edge waves and comments on "A new bending wave solution for the classical plate equation" [J. Acoust. Soc. Am. 104, 2220-2222 (1998)]", J. Acoust. Soc. Am. 107:3 (2000), 1781-1784.

[4] J. B. Lawrie and J. Kaplunov, "Edge waves and resonance on elastic structures: an overview", Math. Mech. Solids 17:1 (2012), 4-16.

[5] J. Cerv, T. Kroupa, and J. Trnka, "Influence of principal material directions of thin orthotropic structures on Rayleigh-edge wave velocity", Comp. Struct. 92:2 (2010), 568-577.

[6] B. S. Vien, L. R. F. Rose, and W. K. Chiu, "Experimental and computational studies on the scattering of an edge-guided wave by a hidden crack on a racecourse shaped hole", Materials 10:7 (2017), 732.

[7] M. V. Wilde, M. V. Golub, and A. A. Eremin, "Experimental and theoretical investigation of transient edge waves excited by a piezoelectric transducer bonded to the edge of a thick elastic plate", J. Sound Vib. 441 (2019), 26-49.

[8] C. J. Chapman, "The structure of rotating sound fields", Proc. R. Soc. London A 440:1909 (1993), $257-271$.

[9] A. N. Norris, "Flexural edge waves", J Sound Vib 171:4 (1994), 571-573.

[10] I. Thompson, I. D. Abrahams, and A. N. Norris, "On the existence of flexural edge waves on thin orthotropic plates", J. Acoust. Soc. Am. 112:5 (2002), 1756-1765.

[11] G. T. Piliposian, M. V. Belubekyan, and K. B. Ghazaryan, "Localized bending waves in a transversely isotropic plate", J. Sound Vib. 329:17 (2010), 3596-3605.

[12] Y. B. Fu, "Existence and uniqueness of edge waves in a generally anisotropic elastic plate", Quart. J. Mech. Appl. Math. 56:4 (2003), 605-616.

[13] M. Destrade, Y. Fu, and A. Nobili. "Edge wrinkling in elastically supported pre-stressed incompressible isotropic plates", Proc. R. Soc. A 472:(2193) (2016), 20160410.

[14] A. S. Zilbergleit and I. B. Suslova, "Contact bending waves in thin plates", Sov. Phys. Acoust. 29 (1983), $108-111$.

[15] M. Destrade and Y. B. Fu, "A wave near the edge of a circular disk", Open Acoust. J. 1 (2008), 15-18.

[16] J. Kaplunov, D. A. Prikazchikov, and G. A. Rogerson, "On three-dimensional edge waves in semi-infinite isotropic plates subject to mixed face boundary conditions", J. Acoust. Soc. Am. 118:5 (2005), 2975-2983. 
[17] V. Zernov and J. Kaplunov, "Three-dimensional edge waves in plates", Proc. R. Soc. A 464:2090 (2008) $301-318$

[18] A. A. Krushynska, "Flexural edge waves in semi-infinite elastic plates", J. Sound Vib. 330:9 (2011) 19641976.

[19] J. Kaplunov, D. A. Prikazchikov, G. A. Rogerson, and M. I. Lashab, "The edge wave on an elastically supported Kirchhoff plate", J. Acoust. Soc. Am. 136:4 (2014), 1487-1490.

[20] J. Kaplunov and A. Nobili, "The edge waves on a Kirchhoff plate bilaterally supported by a two-parameter elastic foundation", J. Vib. Control 23:12 (2017), 2014-2022.

[21] M. Belubekyan, K. Ghazaryan, P. Marzocca, and C. Cormier, "Localised bending waves in a rib-reinforced elastic orthotropic plate", J. Appl. Mech. 74 (2007) 169-171

[22] A. S. Alzaidi, J. Kaplunov, and L. Prikazchikova, "Elastic bending wave on the edge of a semi-infinite plate reinforced by a strip plate", Math. Mech. Solids 24:10 (2019), 3319-3330.

[23] G. Nie and B. Dai, "Bending waves in a semi-infinite piezoelectric plate with edge coated by a metal strip plate", Wave Motion 103 (2021), 102731.

[24] J. Kaplunov and D.A. Prikazchikov, "Explicit models for surface, interfacial and edge waves", pp. 73-114 in Dynamic Localization Phenomena in Elasticity, Acoustics and Electromagnetism, edited by R.V. Craster and J. Kaplunov, Springer, 2013.

[25] J. Kaplunov, D.A. Prikazchikov, and G. A. Rogerson, "Edge bending wave on a thin elastic plate resting on a Winkler foundation", Proc. R. Soc. A 472:2190 (2016), 20160178.

[26] J. Kaplunov and D. A. Prikazchikov, "Asymptotic theory for Rayleigh and Rayleigh-type waves", Adv. Appl. Mech. 50 (2017), 1-106.

[27] S. A. Epshtein, F. M. Borodich, and S. J. Bull, "Evaluation of elastic modulus and hardness of highly inhomogeneous materials by nanoindentation", Appl. Phys. A 119(1) (2015) 325-335.

[28] S. Althobaiti and D. Prikazchikov, "Edge bending waves on an orthotropic elastic plate resting on the Winkler-Fuss foundation", Proc. Nat. Acad. Sci. Armenia 69:1 (2016), 16-24.

[29] I. S. Gradshteyn, and I. M. Ryzhik, Table of integrals, series, and products. Academic Press, San Diego, 2014.

[30] A. Nobili and D. A. Prikazchikov, "Explicit formulation for the Rayleigh wave field induced by surface stresses in an orthorhombic half-plane", Europ. J. Mech. A/Solids 70 (2018), 86-94.

[31] Y. Fu, J. Kaplunov, and D. Prikazchikov, "Reduced model for the surface dynamics of a generally anisotropic elastic half-space", Proc. R. Soc. A 476:2234 (2020), 20190590.

[32] M.A. Siddiqui and M. A. Hawwa, "Flexural edge waves in a Kirchhoff plate carrying periodic edge resonators and resting on a Winkler foundation", Wave Motion 103 (2021), 102720.

[33] B. Ungureanu, M. P. Makwana, R. V. Craster, and S. Guenneau, "Localizing elastic edge waves via the topological rainbow effect", Phys. Rev. Appl. 15:1 (2021), 014057.

Department of Sciences and Technology, Ranyah University Collage, Taif University, P.O. Box 11099, TAIF 21944, SAUDi ARABIA

Email address: snthobaiti@tu.edu.sa

Fini Novo mesto, Faculty of Industrial Engineering Novo mesto, ŠEgova ulica 112, 8000, Novo Mesto, Slovenia

Email address: anatolij.nikonov@fini-unm.si

School of Computing and Mathematics, Keele University, Keele, Staffordshire, ST5 5BG, UK

Faculty of Mechanics and Mathematics, al-Farabi Kazakh National University, Almaty, KazaKHSTAN

Email address: d.prikazchikov@keele.ac.uk 